\title{
Sexual and Psychological Violence Among Pregnant Women in Tigray, Ethiopia
}

\author{
Girmay Adhena ${ }^{1, *}$, Lemessa Oljira ${ }^{2}$ \\ ${ }^{1}$ Department of Reproductive Health, Tigray Regional Health Bureau, Tigray, Ethiopia \\ ${ }^{2}$ School of Public Health, College of Health and Medical Science, Haramaya University, Harar, Ethiopia
}

Email address:

girmayrh@gmail.com (G. Adhena), girmayadhena14@gmail.com (G. Adhena)

${ }^{*}$ Corresponding author

\section{To cite this article:}

Girmay Adhena, Lemessa Oljira. Sexual and Psychological Violence Among Pregnant Women in Tigray, Ethiopia. American Journal of Health Research. Vol. 8, No. 6, 2020, pp. 96-101. doi: 10.11648/j.ajhr.20200806.11

Received: September 19, 2020; Accepted: September 30, 2020; Published: November 4, 2020

\begin{abstract}
Background: Violence against women is a global concern and it is high in Africa particularly in Ethiopia. However, the attention given to this issue is less. This study aimed to assess sexual and psychological violence among pregnant women in Tigray, Ethiopia. Methods: Institutional based cross-sectional survey was done among 476 pregnants. Study participants were selected using a systematic random sampling technique. Pretested, structured, and interview was done using a standardized questioner. Logistic regression analyses (bivariable and multivariable) were done to identify associated factors. The odds ratio of $95 \%$ was used to show the strength and variables with p-value $<0.05$ were considered as statistically significant. Results: About 30.3\% women were experienced sexual and psychological violence. Sexual violence (15.5\%), and psychological violence $(22.7 \%)$. Having alcohol drinker husband [( $\mathrm{AOR}=3.15,95 \% \mathrm{CI}$ : $(1.92,5.18)]$, undesired pregnancy by husband [(AOR=3.68, 95\% CI: $(1.54,8.78)]$, having another wife [(AOR $=4.87,95 \% \mathrm{CI}:(2.07,11.5)]$, unplanned pregnancy [(AOR=3.46, 95\% CI: $(1.51,7.95)]$, low decision making capacity of women [(AOR=2.99, 95\% CI: $(1.85,4.82)]$ and having medium economic status $[\mathrm{AOR}=0.53,95 \% \mathrm{CI}:(0.31,0.92)]$ were significantly associated factors. Conclusion: Near to one third of pregnant women experienced sexual and psychological violence during a recent pregnancy. Strengthening women's, empowerment and encouraging inter-spousal communication to enhance relationships, and to make join reproductive decisions are crucial to reducing violence during pregnancy.
\end{abstract}

Keywords: Sexual Violence, Psychological Violence, Pregnant, Tigray, Ethiopia

\section{Background}

Violence against women is any act of gender-based violence that results in or is likely to result in, physical, sexual, or psychological harm or suffering to women as well as controlling behaviors [1]. It is the most pervasive yet under-recognized human rights violation in the world [2]. In addition to causing injury, it increases women's long-term risk of several other health problems, including chronic pain, physical disability, and depression [1].

Domestic violence ranges from $15 \%$ to $71 \%$ globally [3]. An estimated $30 \%$ of women who have had an intimate partner have suffered violence by their partner [4]. About two-thirds of women exposed to an intimate partner (IPV) at some point in their lives [3, 5]. Prevalence of violence against pregnant women in developing countries is estimated to be 4 to $29 \%$ [6].

A meta-analysis of 92 independent studies concerning domestic violence (DV) among pregnant women showed an average prevalence of $28.4 \%$ emotional abuse and $8.0 \%$ of sexual abuse [7]. The prevalence of intimate partner violence during pregnancy in a study conducted in 19 countries ranged that $2 \%$ to $13.5 \%$ [6]. Domestic violence during pregnancy in less developed countries is about $27.7 \%$ higher than in developed countries (13.3\%) [7]. Violence against women in Africa is a high and systemic review in sub-Saharan Africa showed that $37 \%$ of women were experienced domestic violence with $9.6 \%$ sexual, and $24.6 \%$ psychological violence [8].

Even though Ethiopia ratifies rules and regulations with international agreements to tackle violence against women, 
still it is a major problem in the country [9]. In Ethiopia, studies showed that 50 to $76.5 \%$ of women experienced intimate partner violence in their lifetime [10].

Violence during pregnancy can have serious health consequences for women and their children [4]. Effects such as delayed prenatal care, inadequate weight gain, smoking, substance abuse, sexually transmitted infections (STI), vaginal and cervical infections, kidney infections, miscarriages and abortions, premature labor, fetal distress, and bleeding during pregnancy and mental health problems and poor emotional well-being (emotional distress, depression, anxiety, low self-esteem, and poor attachment) are among the effects of violence during pregnancy [20-23]. Studies revealed that socioeconomic conditions, lower age, lower educational level, not living with the companion, childhood abuse, alcohol abuse and drug abuse by the partner are among the factors that increase the occurrence of violence against women [15].

The majority of studies on intimate partner violence during pregnancy measure physical violence, although sexual and psychological abuse during pregnancy is also considered as detrimental for women and their children's well-being. Thus, this study aimed to assess sexual and psychological violence among pregnants in Ofla, Tigray, Ethiopia.

\section{Methods}

\subsection{Study Area and Period}

The study was conducted in Ofla District, one of the districts in Tigray Regional State, Ethiopia. It is found 619 $\mathrm{km}$ away from Adis Ababa, the capital city of Ethiopia. Based on the 2007 census the District has a total population of 143,745 , out of this number, $69,810(48.6 \%)$ were male and $79,935(51.4 \%)$ were females [16]. The study was conducted from October 1-30, 2019.

\subsection{Study Design and Population}

A facility-based cross-sectional survey was conducted among pregnant women. All pregnants attending ANC in the public health facilities of Ofla were the source population. Pregnant women attending ANC during the data collection period constituted the study population. Pregnants who were unable to interview due to severe medical problems were excluded.

\subsection{Sample Size Calculations and Sampling Procedures}

The sample size was calculated using single population formula $\left(n=z^{2} p(1-p) / d^{2}\right)$, where $(z$ is the normal standard deviation set at 1.96 , confidence level specified at $95 \%$, and margin of error (d) 5\%,10\% nonresponse rate and prevalence of domestic violence (p) $44.5 \%$ from a previous similar study conducted in Abay Chomen [17]. The calculated sample size for this was 379 and by adding $10 \%$ (nonresponse rate) it gives 417 . The sample size was also calculated for factors by using Epi-info version-7 with the assumption of a $95 \%$ confidence interval, $80 \%$ power, $5 \%$ degree of precision, and a $10 \%$ non-response rate. By using this assumption the calculated sample size was 476 . Since the sample size of the second objective (476) was higher than the first objective (417) thus, the final sample was taken 476. All public health facilities were included and population proportion allocation to sample size was done. Pregnants were selected systematically based on their visiting order until the required sample size met.

\subsection{Data Collection Procedure and Measurement Tool}

Standardized and structured WHO Multicountry study questionnaire for assessing women's health and violence was used for data collection [18]. The questionnaire was translated into the local language (Tigrigna) and pretested in 24 respondents ( $5 \%$ of the sample size) at Alamata which is out of the study area. The questioner includes sociodemographic characteristics (age, ethnicity, educational status, religion, residence, economic status), acceptance of IPV by women (argues with him, burns the food, goes without telling him, failing to complete housework and refusal of men's request for sex), reproductive health characteristics (the plan of pregnancy, the desierdness of pregnancy, sexual partner status), Decision-making capacity of women at the hose hold issue (on how to use the money, healthcare, major household purchases, and visiting her family or relatives). The questionnaire has three items of psychological violence (insult, humiliation, intimidation, or scared on purpose), and it also includes three items of sexual violence (forced sex without the consent of the woman, having sex when women do not want, having an unusual type of sex that hurts her). The prevalence of violence is the proportion of pregnant women reporting at least one act of sexual and/or psychological violence during the current pregnancy by her husband. Data were collected by 12 midwives (degree holders) who were all females which creates an opportunity for disclosure of violence by the women. Two public health professionals and the principal investigator supervised the data collection procedure. Two days of training was given to data collectors and supervisors.

\subsection{Data Processing and Analysis}

First, the collected data were coded, checked, and entered into the Epi-data version 4.1 statistical software version and then exported into SPSS version 20 for analysis. Descriptive statistical analyses such as simple frequencies, percentages, measures of central tendency, and measure of variability were used to describe the characteristics of participants and their husbands. Information was presented using narratives, frequencies, summary measures, tables, and figures. The principal component analysis was used to produce wealth quintiles. The bivariable analysis was carried out to see the association of each independent variable with the outcome variable. Variables that have an association with the outcome variable at a p-value of less than 0.25 were selected as candidates for multivariable analysis. The multivariable analysis was performed in the logistic regression up on controlling for the possible confounding factors. Hosmer- 
Lemeshow tests were conducted to test model goodness of fit Odds ratio with $95 \%$ CI were used to show the strength and direction of the associations. Finally, variable with a p-value less than $0.05 \quad(p<0.05)$ was considered statistically significant.

\subsection{Ethical Considerations}

Ethical clearance was secured from Haramaya University College of Health and Medical Sciences Institutional Health Research Ethics Review Committee. A letter of permission was obtained from Ofla district health office. Informed, written, voluntary, and signed consent was obtained from each head of the health facilities, and each participant after clearly informing them about the purpose, risk, and benefit of the study, the interview was conducted in a separate and calm room in each public health facility.

\section{Result}

\subsection{Background and Other Characteristics of Participants}

About 476 respondents were interviewed making a response rate of $100 \%$. The mean age of the participants was 27.5 (SD \pm 6.3 ) years. Near to half, 229 (48.1\%) of the participants were between the age of 25 and 34 years old. The majority, $326(68.5 \%)$ of the participants were rural dwellers, about 441 (92.6\%) were Orthodox Christian and $471(98.9 \%)$ were Tigray in their ethnicity. More than half of the pregnant women, $255(53.6 \%)$ had no formal education, more than one-third $176(37 \%)$ of the participants lived into households with medium wealth quintile and more than half $(53.6 \%)$ were housewives in their occupational status. About $55(11.6 \%)$ women reported that their current pregnancy was unplanned, More than one-third, 161 (33.8\%) of participants had low discussion-making capacity in their household issues, about 324 (68.1\%) participants accept any form of violence by their husband through justifying reasons of men's violent acts. Regarding husbands of pregnant women, near to half $(49.2 \%)$ had no learned formal education, about $18(3.8 \%)$ were smokers and $41(8.6 \%)$ had other sexual partners. More than half $(52.5 \%)$ were alcohol users and $52(10.9 \%)$ of husbands have no interest in the current pregnancy (Table 1).

Table 1. Background characteristics of pregnant women and their husbands, attending ANC at public health facilities in Ofla District, Tigray, Ethiopia, $2019(N=476)$.

\begin{tabular}{llll}
\hline Characteristics & Categories & Frequency & Percentage (\%) \\
\hline \multirow{2}{*}{ Age } & $15-24$ & 166 & 34.9 \\
& $25-34$ & 229 & 48.1 \\
Religion & $35-49$ & 81 & 17 \\
& Orthodox & 441 & 92.6 \\
Ethnicity & Muslim & 35 & 7.4 \\
\multirow{4}{*}{ Residence } & Tigray & 471 & 98.9 \\
& Amhara & 5 & 1.1 \\
\multirow{3}{*}{ Educational status } & Rural & 326 & 68.5 \\
& Urban & 150 & 31.5 \\
& No formal education & 255 & 53.6 \\
& High school and & 116 & 24.4 \\
\hline
\end{tabular}

\begin{tabular}{|c|c|c|c|}
\hline Characteristics & Categories & Frequency & Percentage (\%) \\
\hline \multirow{6}{*}{ Occupational status } & above & & \\
\hline & $\begin{array}{l}\text { Government } \\
\text { employee }\end{array}$ & 38 & 8 \\
\hline & Merchant & 50 & 10.5 \\
\hline & Housewife & 255 & 53.6 \\
\hline & Unemployed & 133 & 27.9 \\
\hline & No formal education & 234 & 49.2 \\
\hline \multirow{2}{*}{$\begin{array}{l}\text { Educational status } \\
\text { of husband }\end{array}$} & Primary school & 132 & 27.7 \\
\hline & $\begin{array}{l}\text { High school and } \\
\text { above }\end{array}$ & 110 & 23.1 \\
\hline \multirow{2}{*}{$\begin{array}{l}\text { Alcohol } \\
\text { consumption } \\
\text { (husband) }\end{array}$} & Drinks daily & 250 & 52.5 \\
\hline & Did not drink & 226 & 47.5 \\
\hline \multirow{2}{*}{$\begin{array}{l}\text { Plan of current } \\
\text { pregnancy }\end{array}$} & Planned & 421 & 88.4 \\
\hline & Unplanned & 55 & 11.6 \\
\hline \multirow{2}{*}{ Age at first marriage } & $<18$ & 184 & 38.7 \\
\hline & $\geq 18$ & 292 & 61.3 \\
\hline \multirow{2}{*}{$\begin{array}{l}\text { Discussion making } \\
\text { capacity }\end{array}$} & High & 315 & 66.2 \\
\hline & Low & 161 & 33.8 \\
\hline \multirow{2}{*}{$\begin{array}{l}\text { Desire for current } \\
\text { pregnancy } \\
\text { (husband) }\end{array}$} & Desired & 424 & 89.1 \\
\hline & Undesired & 52 & 10.9 \\
\hline \multirow{2}{*}{ Marriage ceremony } & Yes & 419 & 88 \\
\hline & No & 52 & 12 \\
\hline \multirow{2}{*}{$\begin{array}{l}\text { Attitudes to } \\
\text { domestic violence }\end{array}$} & Positive (accept) & 324 & 68.1 \\
\hline & $\begin{array}{l}\text { Negative (do not } \\
\text { accept) }\end{array}$ & 152 & 31.9 \\
\hline \multirow{2}{*}{$\begin{array}{l}\text { Having another wife } \\
\text { (husband) }\end{array}$} & No & 435 & 91.4 \\
\hline & Yes & 41 & 8.6 \\
\hline \multirow{3}{*}{$\begin{array}{l}\text { Smoking status } \\
\text { (husband) }\end{array}$} & Non-smoker & 458 & 96.2 \\
\hline & Smoker & 18 & 3.8 \\
\hline & Medium & 176 & 37 \\
\hline \multirow[t]{2}{*}{ Wealth Index } & Low & 155 & 32.6 \\
\hline & High & 145 & 30.4 \\
\hline
\end{tabular}

\subsection{The Prevalence of Sexual and Psychological Violence}

Among 476 interviewed pregnant women, 144 (30.3\%; 95\% CI: $(26 \%, 34.7 \%))$ had experienced at least one act or treat of sexual and/or psychological violence by their husbands during their recent pregnancy. About 22.7\% respondents report psycological violence. out of this, insulted or make her to feel bad to her self $(19.3 \%)$, humuliating infront of others $(4.4 \%)$, and scare or intimediate on purpose $(6.5 \%)$. About $15.5 \%$ of partcipants exprianced sexual violence. From this number, forced sex $(10.1 \%)$, did unwanted sex because of fear to the hasband $(10.7 \%)$, and forced to do something sexual that is degrading or humiliating to her $(2.3 \%)$ were reported.

\subsection{Factors Associated with Sexual and Psychological Violence}

In the binary logistic regression, educational status, employment status, plan of pregnancy, marriage ceremony, drinking status (husband), acceptance of violence, the interest of pregnancy by husband, economic status, smoking status (husband), discussion making capacity, number of Sexual partners (Husband) were associated factors. In the final model, the plan of pregnancy, drinking status, the desiredness of pregnancy, economic status, discussion making capacity, and the number of sexual partners remain the main 
significantly associated factors (Table 2).

Table 2. Factors independently associated with sexual and psychological violence among pregnants in Ofla District, Tigray, Ethiopia, 2019 (N=476).

\begin{tabular}{|c|c|c|c|c|}
\hline Variables & Violence (\%) & No violence (\%) & COR 95\% CI & AOR 95\% CI \\
\hline \multicolumn{5}{|l|}{ Education status } \\
\hline No formal education & $89(34.9)$ & $166(65.1)$ & $1.63(0.98,2.72)$ & $0.80(0.41,1.58)$ \\
\hline Primary school & $29(25)$ & $87(75)$ & $1.01(0.55,1.87)$ & $0.59(0.28,1.27)$ \\
\hline Secondary and above & $26(24.8)$ & $79(75.2)$ & 1 & 1 \\
\hline \multicolumn{5}{|l|}{ Employment status } \\
\hline Merchant & $9(18)$ & $41(82)$ & $0.71(0.25,1.99)$ & $0.53(0.15,1.93)$ \\
\hline Housewife & $79(31)$ & $176(69)$ & $1.45(0.65,3.19)$ & $0.89(0.29,2.73)$ \\
\hline Unemployed & $47(35.3)$ & $86(64.7)$ & $1.76(0.77,4.03)$ & $0.78(0.26,2.40)$ \\
\hline Government employee & $9(23.7)$ & $29(76.3)$ & 1 & 1 \\
\hline \multicolumn{5}{|l|}{ Plan of pregnancy } \\
\hline Unplanned & $42(76.4)$ & $13(23.6)$ & $10.1(5.22,19.6)$ & $3.46(1.51,7.95)^{*}$ \\
\hline Planned & $102(24.2)$ & $319(75.8)$ & 1 & 1 \\
\hline \multicolumn{5}{|l|}{ Marriage ceremony } \\
\hline No & $21(36.8)$ & $36(63.2)$ & $1.40(0.79,2.50)$ & $1.15(0.54,2.45)$ \\
\hline Yes & $125(29.7)$ & $296(70.3)$ & 1 & 1 \\
\hline \multicolumn{5}{|l|}{ Alcohol use } \\
\hline Drinker & $109(43.6)$ & $141(56.4)$ & $4.22(2.72,6.54)$ & $3.15(1.92,5.18)^{* *}$ \\
\hline Non-drinker & $35(15.5)$ & $191(84.5)$ & 1 & 1 \\
\hline \multicolumn{5}{|c|}{ Desiredness of pregnancy (Husband) } \\
\hline Undesired & $40(76.9)$ & $12(23.1)$ & $10.3(5.19,20.3)$ & $3.68(1.54,8.78)^{*}$ \\
\hline Desired & $104(24.5)$ & $320(75.5)$ & 1 & 1 \\
\hline \multicolumn{5}{|l|}{ Smoking status } \\
\hline Smoker & $13(72.2)$ & $5(27.8)$ & $6.49(2.27,18.6)$ & $2.35(0.56,9.96)$ \\
\hline Nonsmoker & $131(28.6)$ & $327(71.4)$ & 1 & 1 \\
\hline \multicolumn{5}{|l|}{ Acceptance of violence } \\
\hline Accepted & $119(36.7)$ & $205(63.3)$ & $2.95(1.82,4.79)$ & $1.51(0.85,2.68)$ \\
\hline Did not accept & $25(16.4)$ & $127(83.6)$ & 1 & 1 \\
\hline \multicolumn{5}{|l|}{ Wealth index } \\
\hline Low & $32(20.6)$ & $123(79.4)$ & $0.33(0.19,0.55)$ & $0.61(0.33,1.11)$ \\
\hline Middle & $48(27.3)$ & $128(72.7)$ & $0.48(0.29,0.76)$ & $0.53(0.31,0.92)^{*}$ \\
\hline High & $64(44.1)$ & $81(55.9)$ & 1 & 1 \\
\hline \multicolumn{5}{|c|}{ Discussion making capacity } \\
\hline Low & $83(51.6)$ & 78 (48.4) & $4.43(2.92,6.72)$ & $2.99(1.85,4.82)^{* *}$ \\
\hline High & $61(19.4)$ & $254(80.6)$ & 1 & 1 \\
\hline \multicolumn{5}{|c|}{ Number of Sexual partners (Husband) } \\
\hline Two and above & $30(73.2)$ & $11(26.8)$ & $7.68(3.73,15.8)$ & $4.87(2.07,11.5)^{* *}$ \\
\hline One & $114(26.2)$ & $321(73.8)$ & 1 & 1 \\
\hline
\end{tabular}

$*=$-value $<0.05 ; * *=$-value $<0.001 ; \mathrm{COR}=$ crud odds ratio; $\mathrm{AOR}=$ adjusted odds ratio.

\section{Discussion}

About 144, 30.3\% (95\% CI: $(26 \%, 34.7 \%))$ of participants experienced sexual and psychological violence in their current pregnancy. Unplanned pregnancy, having a drinker husband, undesired pregnancy by husband, having middle economic status and husbands' sexual partner status were significantly associated factors.

The prevalence $(30.3 \%)$ is in line with the finding from Nepal (27.2\%) [19], Turkey (30\%) [20]. But the finding in this study is higher than from SouaPolo, Brazil (17.6\%) [21], South Africa 15.5\% [22], Namibia (8.1\%) [12]. The possible reason for the discrepancy might be due to the variation in the sample size, sampling method, and study subjects. However, it is lower than from the finding in Nigeria (55\%) [23], Tanzania (65\%) [24], and systemic review in SubSaharan Africa 37\% [8]. The discrepancy might be due to the variation in the definition where others included all types of violence but this study focused only on sexual and psychological violence. The variation could be due to a discrepancy in the definition and duration of the study in which some studies assessed the lifetime occurrence of violence where this study assessed only during a recent pregnancy. The variation could also be due to the difference in the study subjects, and access to information that may increase violence.

Having alcohol drinker husband was 3.15 times higher experienced violence than those their husband did not consume alcohol. This is supported by the finding of systemic review in Ethiopia [11]. The possible reason could be due to the effect of the alcohol content that disturbs the physical and cognitive performance and reducing self-control and judgment that likely increases violence.

Bing unplanned pregnant were 3.46 times more likely to experience violence as compared to planned pregnants. This is consistent with the study conducted in Huletejuensie (Ethiopia) [25]. The reason could be that unintended pregnancy may lead to academic failers, school dropouts and may increase psychological distress that likely increases conflict within the relationship. 
Pregnants with medium income were 53\% less likely to experience violence as compared to those who had a high income. This is consistent with the finding in Mangalore [26], systemic review in Sub Saharan Africa [8]. The possible reason might be due to the fact the unlimited interest of human beings but the access of the limited resources in our day to day activity that may not be in line with the interest of human beings inaddtion to the increase in demand during pregnancy period that may increase the problem.

Those whose husband did not desire the current pregnancy were 3.86 times more likely to experience violence than those whose husband desired the current pregnancy. This is consistent with the study in Huletejuensie [25]. The possible explanation might be due to the pregnancy could be due to sexual violence and restricting family planning choices by the husband that may increase the occurrence of violence.

Women with low discussion making capacity in the household issue were about three times more likely to experience violence compared to those who have low decision capacity. This is consistent with the finding from Mangalore [26], systemic review in Sub Saharan Africa [8], systemic review in Ethiopia [11]. The reason could be due to male-dominated and controlled all activities of the household that did not give the chance for women to involve in their interest. They may do their day to day activities without there interes this may increases the problem.

Pregnant whose husband had another sexual partners were 4.87 times more likely to experience violence compared to those who had one sexual partner. This is supported by the finding from Turkey [20]. The possible reason could be due to the variation in the culture and religious perspectives of the society in which they only permit one to one relationship.

\section{Limitation}

There maybe social desirability bias due to the sensitivity of the violence.

\section{Conclusion}

Overall, about two-thirds of pregnants were experienced sexual and psychological violence during their current pregnancy. Being unplanned pregnancy, having a drinker husband, undesired pregnancy by husband, having middle economic status, and having a husband who had more than one sexual partner, and low decision-making capacity of women were significantly associated factors with violence. Strengthening women's empowerment and encouraging inter-spousal communication to enhance relationships, and to make join reproductive decisions through community awareness and male involvement in sexual and reproductive health issues are important to reduce the problem.

\section{Abbreviations}

\author{
ANC: Antenatal care \\ DV: Domestic Violence \\ IPV: Intimate Partner Violence \\ SPSS: Statistical package for social sciences \\ STI: Sexually Transmited Disease \\ WHO: World Health Organization.
}

\section{Data Availability}

The data used to support the findings of this study are available from the corresponding author upon reasonable request.

\section{Conflicts of Interest}

The authors declare that they have no competing interests.

\section{Funding}

Financial issues for this work were covered by the authors.

\section{Authors' Contributions}

GA was involved in the inception and design of the study, conducted data collection and entry, performed analysis and interpretation of data, wrote the report, and was a major contributor in writing the manuscript. LO Advised and supported from proposal development to the manuscript preparation. All authors read and approved the final manuscript.

\section{Acknowledgements}

We would like to appreciate to Haramaya University. Our heartfelt thanks also extend to the staff of Ofla/Korem District health offices, supervisors, data collectors, and the study participants for their willingness and cooperation in the data collection process.

\section{References}

[1] World Health Organization. Global and Regional Estimates of Violence against Women: Prevalence and Health Effects of Intimate Partner Violence and Non-partner Sexual Violence. 2013.

[2] WHO. Violence and injury prevention: prevention of intimate partner and sexual violence (domestic violence), 2014.

[3] Garcia-Moreno C, Jansen HA, Ellsberg M, et al. Prevalence of intimate partner violence: findings from the WHO multicountry study on women's health and domestic violence. Lancet. 2006; 368 (9543): 1260-1269.

[4] Stockl H, Devries K, Rotstein A, et al. The global prevalence of intimate partner homicide: a systematic review. Lancet 2013; 382 (9895): 859-865. 
[5] World Health Organization (CH). Understanding and addressing violence against women: intimate partner violence [Internet]. Geneva; 2012 [citado 2015 mar 18]. Available at: http://apps.who.int/iris/bitstream/10665/77432/1/WHO_RHR_ 12.36_eng.pdf.

[6] Devries KM, Kishor S, Johnson H, Stöckl H, Bacchus L, Garcia-Moreno $\mathrm{C}$, et al. Intimate partner violence during pregnancy: prevalence data from 19 countries. Reprod Health Matters. 2010; 18 (36): 1-13.

[7] James L, Brody D, Hamilton Z. Risk factors for domestic violence during pregnancy: a meta-analytic review. Violence Vict. 2013; 28 (3): 359-80.

[8] Bright Opoku Ahinkorah1, Kwamena Sekyi Dickson, and Abdul-Aziz Seidu. Women decision-making capacity and intimate partner violence among women in sub-Saharan Africa. Archives of Public Health. 2018; 76: 5.

[9] EDHS. Central Statistical Agency; Ethiopian Demographic Health Survey Addis Ababa, Ethiopia and Rockville, Maryland, USA: CSA and ICF. 2016.

[10] O’Doherty L, Hegarty K, Ramsay J, Davidson LL, Feder G, Taft A. Screening wom $\neg$ en for intimate partner violence in healthcare settings. Cochrane Database Syst Rev. 2015; 7: CD 007007. doi: 10.1002/14651858.CD007007.pub3.

[11] Agumasie Semahegn and Bezatu Mengistie. Domestic violence against women and associated factors in Ethiopia; a systematic review. Reproductive Health. 2015; 12: 78.

[12] Bikinesi LT, Mash R, Joyner K. Prevalence of intimate partner violence and associated factors amongst women attending antenatal care at Outapi clinic, Namibia: A descriptive survey. African Journal of Primary Health Care \& Family Medicine. 2017; 9 (1), a1512.

[13] E. Fekadu, G. Yigzaw, K. A. Gelaye et al., "Prevalence of domestic violence and associated factors among pregnant women attending antenatal care service at University of Gondar Referral Hospital, Northwest Ethiopia," Biomed Central Women's Health, vol. 18, pp. 1-8, 2018.

[14] B. H. Gebrezgi, M. B. Badi, E. A. Cherkose, and N. B. Weldehaweria, "Factors associated with intimate partner physical violence among women attending antenatal care in Shire Endaselassie town, Tigray, northern Ethiopia: a crosssectional study," Reproductive Health, vol. 14, no. 1, p. 76, 2017.

[15] Jesmin SS. Social determinants of married women's attitudinal accep $\neg$ tance of intimate partner violence. J Interpers Violence. 2015 Aug 5. doi: $10.1177 / 0886260515597436$.
[16] Central Statistical Agency [Ethiopia] and ORC Macro. Ethiopian demographic health survey. Maryland: Ethiopia and Calverton. 2007.

[17] Abate BA, Wossen BA, Degfie TT. Determinants of intimate partner violence during pregnancy among married women in Abay Chomen district, Western Ethiopia: a community-based cross-sectional study. BMC Women's Health. 2016; 16: 16.

[18] García-Moreno C, Jansen H, Ellsberg M, L. Heise, Watts CH. WHO multi-country study on women's health and domestic violence against women: initial results on prevalence, health outcomes and women's responses. 2005.

[19] Monika Shrestha, Sumina Shrestha and Binjwala Shrestha. Domestic violence among antenatal attendees in a Kathmandu hospital and its associated factors: a cross-sectional study. BMC pregnancy and Child Health. 2016, 16: 360.

[20] Selma Sen, and Nursen BolsoyViolence against women: prevalence and risk factors in Turkish sample. BMC Women's Health (2017) 17: 100

[21] Lisiane Camargo Quialheiro de Oliveira, Mariana de Oliveira Fonseca-Machado, Juliana Stefanello, Flávia Azevedo GomesSponholz. Intimate partner violence in pregnancy: identification of women victims of their partners. 2015: 36: 233-8.

[22] Sally Field, Michael Onah, Thandi van Heyningen 1 and Simone Honikman. Domestic and intimate partner violence among pregnant women in a low resource setting in South Africa: a facility-based, mixed methods study. BMC Women's Health (2018) 18: 119.

[23] Adriel Monkam Tchokossa, Timothy Golfa, Omowumi Romoke Salau, Adesola Adenike Ogunfowokan, Perceptions and Experiences of Intimate Partner Violence among Women in Ile-Ife Osun State Nigeria. International Journal of Caring Sciences, 2018, 11; 1; 267.

[24] Method R. Kazaura1, Mangi J. Ezekiel, and Dereck Chitama. Magnitude and factors associated with intimate partner violence in mainland Tanzania. BMC Public Health. 2016: 16: 494.

[25] Tenaw Y, Tesfaye G, gudina E, habtamu M. Magnitude of Domestic Violence and Associated Factors among Pregnant Women in Hulet Ejju Enessie District, Northwest Ethiopia. Hindawi Publishing Corporation. 2014; 2014: 8.

[26] Shiny Chrism Queen Nesan, Gundmi Rakesh Maiya, Rashmi Kundapur. Domestic violence patterns and its consequences among married women in rural Mangalore. Indian Journal of Community Health, 2018; 30. 